\title{
Methanol Exposure Interferes with Morphological Cell Movements in the Drosophila Embryo and Causes Increased Apoptosis in the CNS
}

\author{
Dervla M. Mellerick, ${ }^{*}$ Heather Liu \\ Department of Pathology, University of Michigan Medical Center, Ann Arbor, Michigan 48109
}

Received 7 November 2002; accepted 24 November 2003

\begin{abstract}
Despite the significant contributions of tissue culture and bacterial models to toxicology, whole animal models for developmental neurotoxins are limited in availability and ease of experimentation. Because Drosophila is a well understood model for embryonic development that is highly accessible, we asked whether it could be used to study methanol developmental neurotoxicity. In the presence of $\mathbf{4 \%}$ methanol, approximately $35 \%$ of embryos die and methanol exposure leads to severe CNS defects in about half those embryos, where the longitudinal connectives are dorsally displaced and commissure formation is severely reduced. In addition, a range of morphological defects in other germ layers is seen, and cell movement is adversely affected by methanol exposure. Although we did not find any evidence to suggest that methanol exposure affects the capacity of neuroblasts to divide or induces inappropriate apoptosis in these cells, in the CNS of germ band
\end{abstract}

retracted embryos, the number of apoptotic nuclei is significantly increased in methanol-exposed embryos in comparison to controls, particularly in and adjacent to the ventral midline. Apoptosis contributes significantly to methanol neurotoxicity because embryos lacking the cell death genes grim, hid, and reaper have milder CNS defects resulting from methanol exposure than wild-type embryos. Our data suggest that when neurons and glia are severely adversely affected by methanol exposure, the damaged cells are cleared by apoptosis, leading to embryonic death. Thus, the Drosophila embryo may prove useful in identifying and unraveling mechanistic aspects of developmental neurotoxicity, specifically in relation to methanol toxicity. ๑ 2004 Wiley Periodicals, Inc. J Neurobiol 60: 308-318, 2004

Keywords: neurotoxicity; model organism; teratogen; screening tool; toxicology

\section{INTRODUCTION}

Neurotoxins have been implicated in the initiation and progression of adult neurodegenerative diseases, such as motor neuron and Parkinson's disease (for review see Dauer and Przedborski, 2003), yet the effects of

* Present address: 8301 B, MSRB III, 1150 West Medical Center Drive, Ann Arbor, MI 48109-0646.

Correspondence to: D.M. Mellerick (dervlam@umich.edu).

Contract grant sponsor: NSF (H.L.).

Contract grant sponsor: NIH.

Contract grant number: RO3 ES 010158.

(C) 2004 Wiley Periodicals, Inc.

Published online 7 June 2004 in Wiley InterScience (www. interscience.wiley.com).

DOI $10.1002 /$ neu. 20020 neurotoxins on fetal development are not well characterized. Teratogens are a leading cause of abnormal development in utero and, as such, can cause miscarriage, birth defects, and many other complications. Pesticides, solvents, heavy metals, biological contaminants, electromagnetic fields, either alone or in combination are all candidate teratogens. Although the teratogenic effects of alcohol consumption, tobacco, and other drugs are relatively well understood, the effects of many unclassified toxins and mixtures of toxins on the developing fetus have not been fully investigated, due in part to lack of suitable model systems. Available information regarding teratogenicity of many chemicals is often limited or of a highly technical nature and difficult to interpret. More sig- 
nificantly, few models are available to identify specific teratogens, such as developmental neurotoxins. The Ames test (Ames et al., 1973) is valuable for screening environmental carcinogens, but does not address specific effects on the developing tissues, such as the nervous system. The complexity and inaccessibility of the vertebrate embryo makes rapid screening for neurotoxins impractical, thus more experimentally accessible organisms are needed. As a model for developmental neurotoxicity, the fruit fly, Drosophila, has many advantages, such as its relative simplicity and ex utero development. Although morphologically quite different, the insect and vertebrate nervous systems express common genes at parallel stages of development (Arendt and Nubler-Jung, 1999; Cornell and Von Ohlen, 2000). Despite clear differences in the overall assembly of the CNS in flies and man, many important regulatory cascades specifying neural patterning and neuronal cell-type differentiation are evolutionarily conserved (for review see Arendt and Nubler-Jung, 1999). Thus, the Drosophila embryo could be a very useful model for studying developmental neurotoxicity.

This study focuses on the neurotoxic effects of methanol in the Drosophila embryo. Methanol's role as a developmental neurotoxin is not adequately understood, although methanol intoxication in adult humans can lead to permanent visual damage and death (Liu et al., 1999). Currently, industrial uses of methanol are expanding, particularly as both an antifreeze and a solvent. Methanol is also a significant byproduct of artificial sweeteners. Methanol was the chemical with the highest release to the environment (air, water, and land) in the 1992 Toxic Release Inventory of 23,630 facilities (EPA, TRA report, 1992). Serum values have been shown to be as high as 2.6 $\mathrm{mg} / \mathrm{L}$ in people exposed to methanol vapor. Exposure to methanol can occur from paint strippers, aerosol spray paints, and engine fuel. In certain countries methanol is used as an alternative to petroleum as engine fuel. Methanol can enter the body by inhalation of contaminated air, consumption of contaminated water, and through direct skin contact. The incidence of accidental exposure of pregnant women to this chemical is likely to increase with its expanding role. Thus, a fuller understanding of the adverse effects of this potential developmental neurotoxin is required.

To increase the likelihood that significant developmental defects would result from exposure to methanol, we initially identified concentrations that caused substantial embryonic lethality. We found that methanol is highly toxic to Drosophila embryos: $1 \%$ methanol caused approximately $10 \%$ embryonic mortality.
Morbidity increases to $35-40 \%$ in the presence of $4 \%$ methanol and about half those embryos have severe CNS defects. By comparing the distribution of cellspecific markers in unexposed and methanol-exposed embryos, we found that ventral midline defects were most commonly associated with methanol exposure. The phenotypes observed in embryos undergoing germ band extension suggested that cell movement associated with gastrulation and germ band extension is particularly sensitive to methanol exposure. We found that the level of bromodeoxyuridine (BrdU) incorporation was not significantly affected in neural precursors exposed to methanol, which suggests that methanol does not affect their division. Analyses of embryos lacking the cell death genes grim, head involution defective (hid), and reaper in conjunction with TUNEL staining indicated that methanol exposure increases the level of programmed cell death, particularly in mesectodermal cells. Thus, apoptosis significantly contributes to the late CNS defects resulting from methanol exposure. This study suggests that the fruit fly embryo may prove to be useful as a screening tool for developmental neurotoxins and for identifying the molecular mechanisms underlying the action of certain neurotoxins.

\section{MATERIALS AND METHODS}

\section{Flies, Embryo Collections, TUNEL Analysis, BrdU Labeling, and Immunohistochemistry}

Wild-type, Oregon R, flies were used. Df(3L)H99 flies, with a small deficiency at the 35C1-2 location that deletes the grim, head involution defective (hid), and reaper genes (White et al., 1994) were obtained from the Bloomington Stock Center. Embryos were collected following direct deposition and aging on cotton wool soaked in yeast containing $0,1,2$, or $4 \%$ methanol (which sat on grape juice agar). Alternatively, a $1 \mathrm{~h}$ collection of embryos was aged on cotton wool soaked in yeast containing various concentrations of methanol. Thus, unless otherwise stated in the text, embryos were exposed to methanol throughout their development. TUNEL analysis was performed according to Sweeney et al. (2000) using the Dead End kit (Promega). Briefly, embryos were fixed with heptane saturated with $4 \%$ paraformaldehyde (para) in PBS, dechorionated quickly with methanol, and immediately transferred to PBS containing $0.1 \%$ Triton X-100 (PBT). They were digested with 2.5 $\mu \mathrm{g} / \mathrm{mL}$ of proteinase $\mathrm{K}$ for $1 \mathrm{~min}$. The digestion was stopped with glycine, and embryos were fixed a second time with $4 \%$ para in PBS. Embryos were washed for $30 \mathrm{~min}$ with three solution changes with PBT and then once with terminal transferase buffer (Promega). Labeling reaction (100 $\mu \mathrm{L}$; Dead End Kit; Promega) was used per $40 \mu \mathrm{L}$ of 
embryos. The tubes were sealed with Parafilm and incubated in a rotating waterbath for $3 \mathrm{~h}$ at $37^{\circ} \mathrm{C}$. The Vectastain $\mathrm{ABC}$ kit (Vector Labs) was used to detect labeled nuclei. Note that we found that paraformaldehyde fixation was critical for TUNEL staining.

BrdU labeling was performed as described by Shermoen (2000). Following dechorionation in 50\% bleach, embryos were washed with water extensively. They were then permeabilized with octane for $4 \mathrm{~min}$ with constant agitation. Following octane evaporation, embryos were labeled with 1 $\mathrm{mg} / \mathrm{mL}$ BrdU (Sigma) in PBS for $7.5 \mathrm{~min}$. Then they were washed with PBS and fixed in $4 \%$ formaldehyde in PBS. After fixation, they were stored in methanol at $-20^{\circ} \mathrm{C}$. Prior to antibody incubation, the embryonic DNA was denatured with $2.2 \mathrm{~N} \mathrm{HCl}$ containing $0.1 \%$ Triton $\mathrm{X}-100$ by rocking at room temperature for $30 \mathrm{~min}$, with one solution change. Embryos were then neutralized with $0.1 M$ borax (Sigma) for $5 \mathrm{~min}$. After extensive rinsing with PBT, embryos were processed for immunohistochemistry as previously described (Mellerick et al., 1992) using a 1/10 dilution of an anti-BrdU monoclonal antibody (George-Weinstein et al., 1993). Immunohistochemistry was performed as previously described (Mellerick et al., 1992). The following primary antibodies were used: Mouse anti 22c10, 1:10 (Goodman et al., 1984); rabbit anti-Repo 1:200 (Halter et al., 1995); mouse anti-BP102, 1:10 (Patel et al., 1989); mouse antiEngrailed 1:5 (Patel et al., 1989); rat anti-Even-skipped, 1:2000 (Frasch et al., 1987); mouse anti-Slit 1:10 (Rothberg et al., 1990); rabbit anti-Twist, 1:200 (Thisse et al., 1988). The Vectastain Standard kit (Vector Labs) with a peroxidase-conjugated secondary antibody was used to detect antibody binding. All primary and secondary antibodies were preabsorbed as described previously (Mellerick et al., 1992).

\section{RESULTS}

\section{Late CNS Defects in Methanol-Exposed Embryos}

During the course of evaluating the toxic effects of polychlorinated biphenyls (PCBs), a family of aromatic hydrocarbons, on Drosophila embryonic neural development, we inadvertently discovered that methanol was highly toxic to the developing embryo. Methanol was the solvent for the PCBs. The Drosophila embryo is very insensitive to Araclor (a complex mixture of PCBs), with concentrations greater than 1000 PPM required to induce significant embryonic lethality and CNS phenotypes (data not shown). In contrast, we found that $1 \%$ methanol caused approximately $10 \%$ percent embryonic lethality, relative to $100 \%$ viability when water was included as a control. When the levels of methanol were raised to $4 \%$, lethality was close to $40 \%$. Because the defects observed were similar in embryos exposed to methanol concentrations between 1-4\% dur-
Table 1 CNS Axonal Defects in Drosophila Embryos Resulting from Exposure to 4\% Methanol throughout Development

\begin{tabular}{lc}
\hline Defect & Frequency \\
\hline Disrupted commissures in one or two segments & $18 \%$ \\
Absence of, or disruption in, commissures in & \\
$\quad$ more than three segments & $9 \%$ \\
Interrupted longitudinal connectives & $21 \%$ \\
Laterally misplaced longitudinal connectives & $18 \%$ \\
\hline
\end{tabular}

Embryos were scored for microscopically identifiable CNS defects, $n=150$. Three of the defects above were most commonly seen $(50 \%)$, one defect at $25 \%$ frequency, and two at $25 \%$ frequency.

ing development, with the severity correlating with the methanol concentration, we focus here on embryos exposed to $4 \%$ methanol. Methanol exposure during embryogenesis also results in larval and pupal death. In addition to those embryos that failed to emerge from the egg case as a result of exposure to $4 \%$ methanol during embryogenesis, we found that an additional $20 \%$ ( $n$ $=350$ ) of larvae died prior to adulthood, despite the fact that they went through instar larval and pupal development under normal conditions. Although not studied in detail, adult flies when exposed to methanol were agitated, had reduced climbing ability, and reduced egg laying.

To address whether the embryonic CNS is affected by methanol exposure, we compared the distribution of the BP102 antigen that is expressed on CNS axons in control and methanol-exposed embryos. Embryos were directly deposited on yeast soaked cotton wool with/without methanol and developed under those conditions. About $20 \%$ of embryos exposed to methanol throughout embryogenesis presented CNS defects that were identifiable at a gross level using the BP102 antibody. Commissural defects and lateral displacement of the longitudinal connectives were typically observed following methanol exposure during development. Table 1 summarizes the types and range of CNS defects observed, while Figure 1 shows examples of the defects seen.

Next, we examined embryos that were exposed to methanol constantly during embryogenesis for more specific CNS defects, by staining them with antibodies that recognize additional neuronal and glial-specific antigens and comparing antigen distribution to that in control embryos (Fig. 2). Figure 2 compares the distribution of the $22 \mathrm{C} 10$ Futsch antigen, which is normally expressed on pioneering neurons [Fig. 2(A)], Even-skipped (Eve), which is expressed in a segmentally repeated pattern of midline proximal $\mathrm{aCC} / \mathrm{pCC}$ and RP2 neurons, and the more laterally 

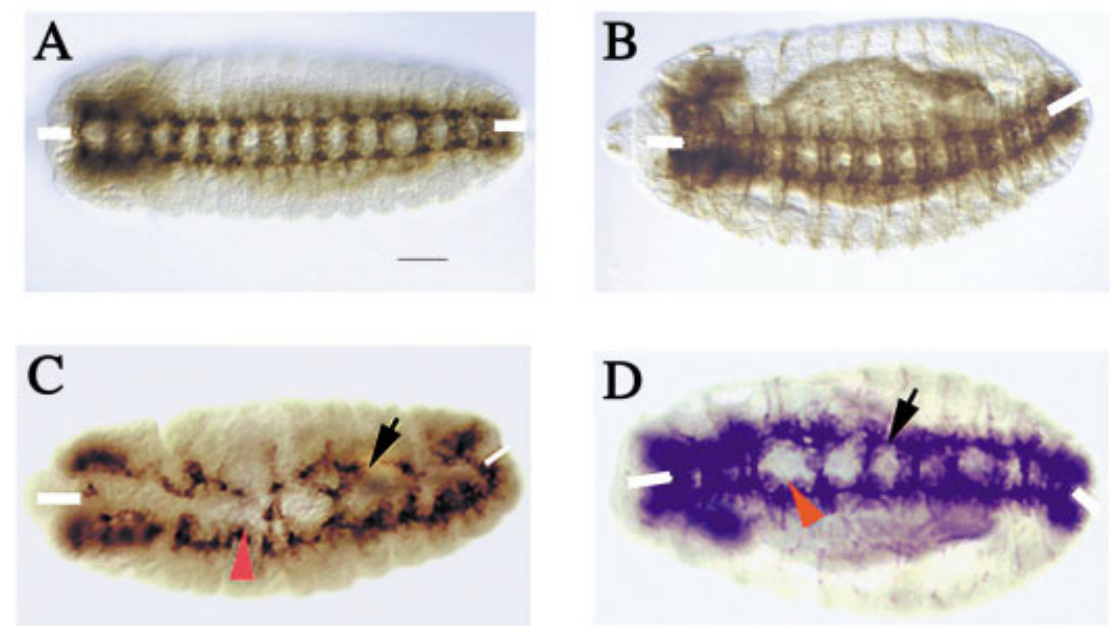

Figure 1 CNS defects seen in Drosophila embryos exposed to methanol during development. Dissected CNSs of BP102 stained embryos are shown; anterior is to the left. The size bar is $50 \mu \mathrm{m}$. The midline is highlighted with a white bar. (A) and (B) Under normal developmental conditions, BP102 is expressed on commissural neurons in an X arrangement in early stage 13 embryos, and then in the anterior and posterior commissures, once they have separated. The bilateral longitudinal connectives, which run anterio-posteriorly, also express the antigen. (C) and (D) Examples of stage 13 (C) and stage 14 (D) embryos that developed in the presence of $4 \%$ methanol. The longitudinal connectives are disrupted and laterally displaced (arrows), and commissural formation is severely affected.

positioned U and EL neurons [Fig. 2(E)], and Engrailed (En), which is expressed in the medially positioned progeny of the midline neuroblast and in clusters of lateral neurons [Fig. 2(G)]. Repo and Slit distribution was also compared in methanol-exposed embryos and controls. Repo is expressed in all glia other than the midline glia [Fig. 2(C)], while Slit is a midline-glial marker [Fig. 2(I); Rothberg et al., 1990]. The aberrant distribution of the CNS specific antigens in methanol-exposed embryos reveals a common pattern. Midline, and midline-adjacent, cells that normally express the various antigens, either do not express them or express the antigen but in cells located lateral to the normal position of those cells that normally express it [Fig. 2(B,D,F,H)]. These observations suggested that midline cells died or changed their identity. When we examined the distribution of the midline glial marker, Slit, in control and methanol-exposed embryos we found that this was the case. In the example shown in Figure 2(J), less Slit-positive are seen in the methanol-exposed embryo compared to the control [Fig. 2(I)], and they are abnormally laterally positioned relative to the organized control pattern. This pattern is highly reproducible. Slit-positive cadavers can be seen in phagocytes overlying the CNS [Fig. 2(J), inset], which strongly suggests that at least some midline cells die inappropriately due to methanol exposure.

\section{Morphological Cell Movements Are Affected by Methanol Exposure}

When embryos were allowed to develop under normal conditions until stage 11 and were then exposed to methanol, the majority had a relatively normal CNS axonal scaffold (95\%), although approximately $15 \%$ of these had a very mildly axial twist [Fig. 2(B)], while commissural defects were seen in one to three segments in less than $2 \%$ of the embryos [e.g., Fig. 2(C)], and the longitudinal connectives were positioned normally relative to each other. These observations suggest that most CNS defects seen in methanol-exposed embryos likely result from disruptions in earlier developmental processes. To address this possibility we compared the distribution of a variety of markers in embryos between stages $8-11$, the time when the germ band is extending. Table 2 summarizes the defects that were most commonly observed. Figure 3 shows examples of affected embryos that are stained with an antibody against Engrailed, which is expressed in a segmentally repeated pattern of anterior-posterior stripes initially in ectodermal cells, and then in CNS neuroblasts and their progeny (Carroll et al., 1988). The altered distribution of this transcription factor in methanol-exposed embryos identifies the defects most commonly resulting from methanol exposure. These include ventral midline defects, anteri- 

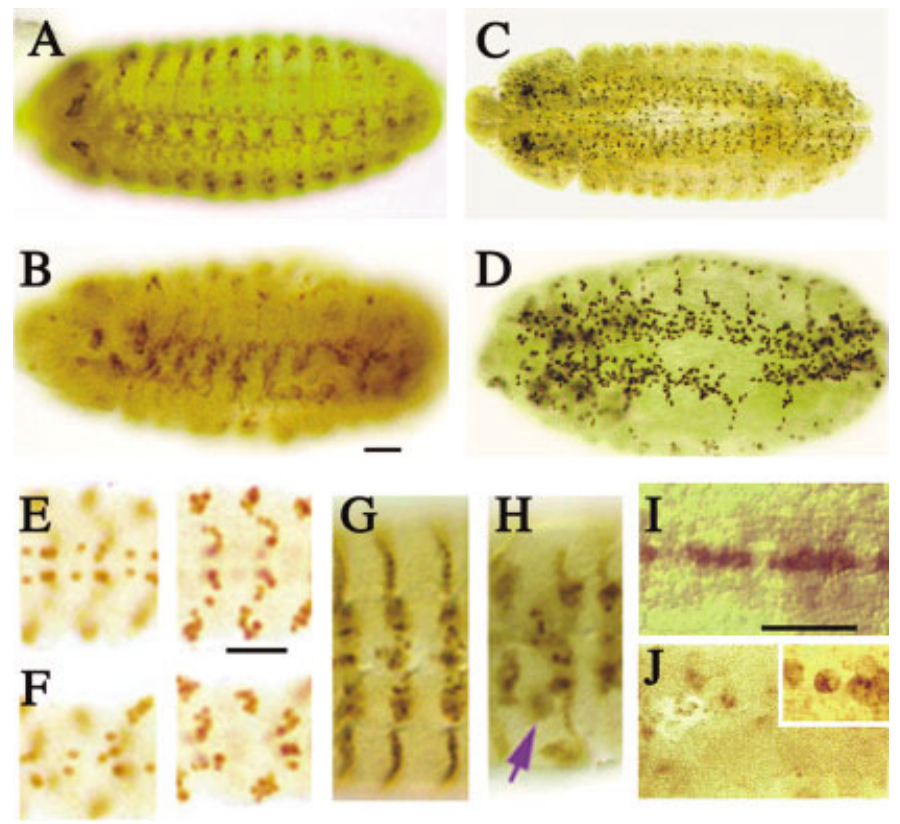

Figure 2 Altered distribution of neuronal and glial markers in the CNS of methanol-exposed embryos. Immunohistochemical distribution of 22C10 (A,B), Repo (C,D), Eve (E,F), En (G,H), and Slit $(\mathrm{I}, \mathrm{J})$ in control $(\mathrm{A}, \mathrm{C}, \mathrm{E}, \mathrm{G}, \mathrm{I})$ and methanol-exposed $(\mathrm{B}, \mathrm{D}, \mathrm{F}, \mathrm{H}, \mathrm{J})$ embryos. Anterior is to the right, and views are ventral. (E) and (F) show the dissected CNS; all others are whole mount presentations. The size bar is $50 \mu \mathrm{m}$. (A) In stage 13 embryos, 22C10, which recognizes the Futsch antigen, is expressed by the midline VUMs, interneurons in a repetitive overlapping 8-type arrangement, and motor neurons exciting the CNS. (B) 22C10 distribution in a mildly affected embryo that developed in the presence of $4 \%$ methanol, showing defects in motor neurons and interneurons. (C) Repo is expressed in all glia, apart from the midline glia. The glia are in a triangular arrangement in each hemi-segment, bordering the ventral midline. (D) In the abdominal segments of a severely affected methanol-exposed embryo, the pattern of glial distribution is disrupted, and the cells are located dorsal to their normal position. (E) Eve is expressed in the aCC, pCC, and RP2 midline-adjacent neurons (dorsal field of focus; left panel) and the laterally positioned $U$ and EL neurons (ventral field of view; right panel). (F) In this moderately affected embryo, the midline proximal aCC, pCC, and RP2 neurons are either aberrantly positioned or missing (left panel); the number of laterally positioned neurons is relatively normal, but they are abnormally positioned (right panel). (G) En is expressed by neurons in the posterior of each segment including the midline neuroblast progeny, and bilateral cluster of neurons. $(\mathrm{H})$ The En positive neurons are inappropriately positioned; the arrow points to an ectopic En lateral cluster. (I) Slit, the Roundabout ligand, is expressed by midline glia in a segmentally repeated pattern. (J) In this severely affected embryo, a gap is seen in the midline and the Slit-expressing glia are scattered about the gap. Overlying this field of view, Slit-expressing cadavers can be seen in phagocytes (inset).

or-posterior segmentation defects, ectopic lateral furrows, and a twisted or lob-sided germ band. One or more of these defects was seen in approximately $40 \%$ of embryos that developed in the presence of $4 \%$ methanol (see Table 2). In addition, random defects were seen in lateral CNS and ectodermal cells, although at a much lower frequency. The morphological abnormalities observed suggest that cell movements, during gastrulation and/or germ band extension, are adversely affected by methanol (Fig. 4).

To further assess whether gastrulation is affected by methanol, we examined the distribution of the
Table 2 Morphological Defects in Stages 8-11 Drosophila Embryos That Were Exposed to 4\% Methanol from Deposition until Collection

\begin{tabular}{lc}
\hline \multicolumn{1}{c}{ Defect } & Frequency \\
\hline Ventral midline defects & $16 \%$ \\
Ectopic furrows & $8 \%$ \\
Nonsymmetrical furrows & $4 \%$ \\
Twisted germ band & $15 \%$ \\
Head defects & $5 \%$ \\
Segmentation defects & $5 \%$ \\
Aberrant cell-specific gene expression & $10 \%$ \\
\hline
\end{tabular}



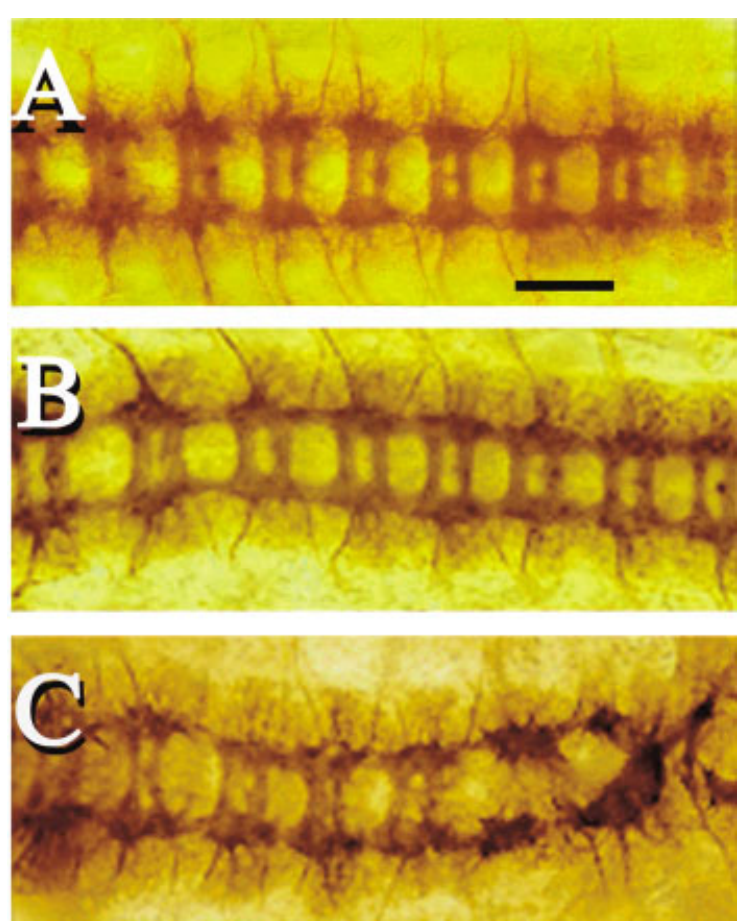

Figure 3 CNS defects in embryos exposed to methanol after stage 11. The CNS of a control embryo (A) and two embryos that developed under normal conditions until stage 11, after which they were exposed to $4 \%$ methanol until their collection [(B) and (C)]. Views are ventral, with anterior to the right. The size bar is $50 \mu \mathrm{m}$. (A) BP102 is expressed on the bilateral longitudinal connectives, the anterior and posterior commissures, the VUM fascicle extending between the commissures, and the intersegmental and segmental nerves. (B) Most methanol-exposed embryos have a relatively normal CNS. (C). In less than $2 \%$, commissure formation is affected in one to two segments, whereas the longitudinal connectives are relatively normal.

transcription factor, Twist, in younger embryos that were exposed to methanol since their deposition. Under normal developmental conditions, Twist is expressed in mesodermal precursor cells before (not shown) and during gastrulation [Fig. 5(A)], and in the mesoderm once gastrulation is completed [Fig. 5(B)]. In less than $5 \%$ of embryos completing gastrulation in the presence of methanol, the dorsal boundaries of Twist expression were uneven compared to the wildtype distribution of the protein [e.g., Fig. 5(C)]. In an additional $10 \%$ of embryos undergoing germ band extension, the mesoderm was asymmetrically positioned [e.g., Fig. 5(D)], in comparison to its position in embryos that develop under normal conditions. These observations suggest that both gastrulation and germ band extension are sensitive to methanol exposure.

\section{Methanol Exposure Does Not Significantly Affect Neuroblast Division, but Induces an Apoptotic Response in Older Drosophila Embryos}

The neurotoxic effects observed in methanol-exposed embryos appear to be due, at least in part, to cell movement and/or identity being affected, which likely results in premature and/or inappropriate cell death. However, methanol exposure could also interfere with normal cell division. To enable us to explore these possibilities, we performed BrdU incorporation and TUNEL assays.

Within the developing CNS, BrdU is extensively incorporated into dividing neuroblasts in embryos undergoing germ band extension. We found that the overall levels of BrdU incorporation were similar in normal and methanol-exposed embryos until the middle of stage 12 (data not shown). In late stage 12 embryos, when the germ band is completing retraction, we found that BrdU positive neuroblasts and ganglion mother cells are inappropriately positioned at either side of an expanded ventral midline, and there was an overall decrease in the level of BrdU incorporation associated with methanol exposure following germ band retraction (Fig. 6 and data not shown). Thus, cell division is affected by methanol typically only during and after germ band retraction. The anomalous distribution of dividing cells at the terminal stages of germ band retraction correlates with the CNS phenotypes that we observe resulting from methanol exposure (see Fig. 1). Because we failed to see such inappropriate positioning of ventrolateral cells prior to this stage in development, we hypothesized that an apoptotic response may be induced in stage 12 and older embryos that may contribute to the CNS phenotypes that we observed in embryos that developed in the presence of methanol.

\section{Apoptosis Contributes Significantly to the Late CNS Defects Seen in Methanol- Exposed Embryos}

Apoptosis or programmed cell death is induced by a variety of insults in both Drosophila and vertebrates, and occurs normally during the later stages of embryonic development (Abrams et al., 1993). Normally, prior to germ band retraction, few apoptotic cells are seen in the germ band region of the embryo. Apoptosis is limited primarily to the developing brain until the middle of stage 12 . In less than $0.5 \%$ of methanolexposed embryos inappropriate cell death was seen before mid stage 12 (data not shown). In contrast, from late stage 12 onwards, we found a significant 

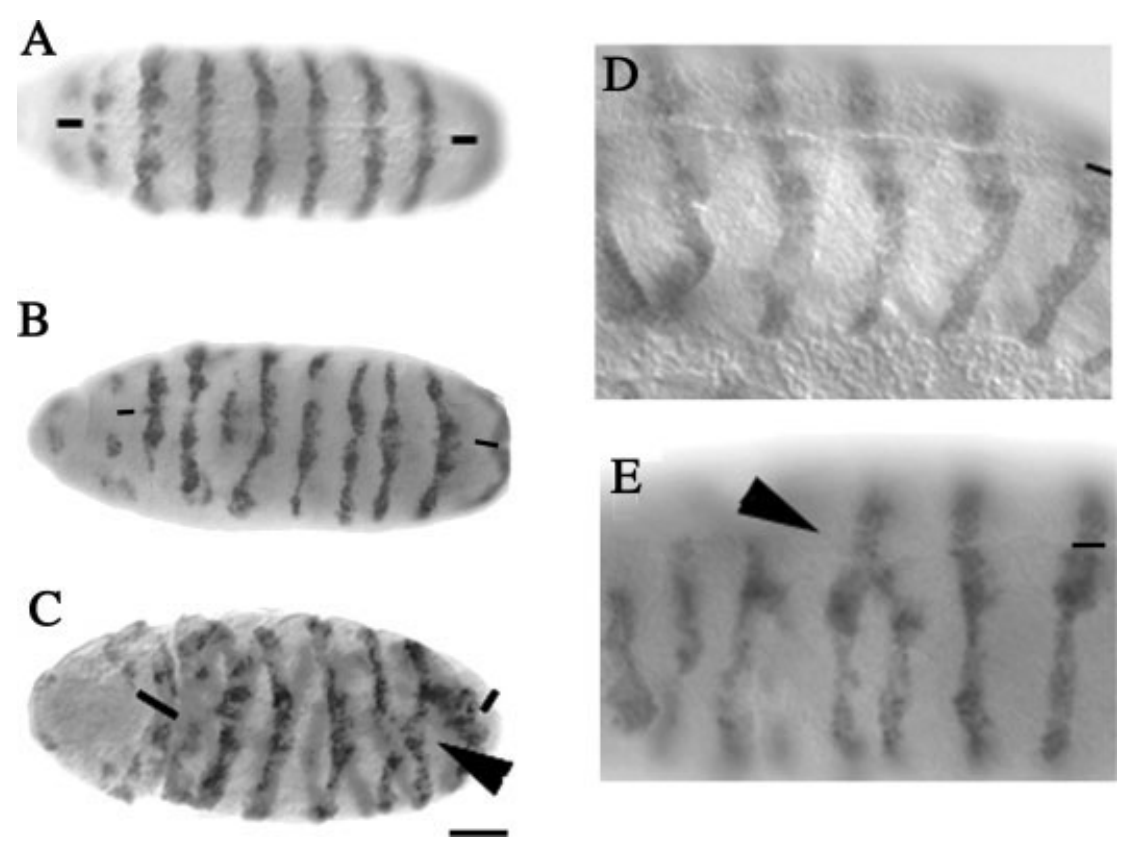

Figure 4 Defects in embryos undergoing germ band extension resulting from methanol exposure. Immunohistochemical distribution of Engrailed (En) in control embryos [(A) and (D)]; (B), (C), and (E) are methanol-exposed embryos. Views are ventral (A-C) and lateral [(D) and (E)]. Anterior is to the right. The size bar is $50 \mu \mathrm{m}$. The position of midline is indicated by a black bar. (A) Normally, in stage 9 embryos, En is expressed in a segmentally repeated pattern of anterior-posterior stripes in midline adjacent neuroblasts and in the lateral ectoderm. (B) This methanol-exposed embryo has a slightly twisted germ band, and the En stripes are disrupted in the thoracic segments of the embryo. (C) This embryo has a more exaggerated twist of the germ band, and the En stripes in two abdominal segments are fused at the ventral midline (arrowhead). (D) The rows of En-expressing cells are in a regular pattern in control embryos. (E) In two segments of the methanol-exposed embryos shown, the En stripes are fused at the ventral midline (arrowhead), and the pattern of lateral En expressing neuroblasts and ectodermal cells is disrupted.

increase in the level of apoptosis in CNS cells, particularly those in, and close to, the ventral midline in methanol-exposed embryos relative to controls. Although the degree of excess apoptosis varied among embryos, the levels were close to $20 \%$. Figure 7 shows examples of methanol-exposed embryos with increased distribution of apoptotic cells. Normally apoptotic cells are limited to the posterior of the germ band and at low levels in the lateral regions of the CNS in stage 13 embryos [Fig. 7(A)]. In addition, a subset of midline cells normally die during late stage 12 and stage 13 (for review see Jacobs, 2000). In stage 13 methanol-exposed embryos, localized groups of apoptotic cells were seen in the ectoderm overlying the CNS [Fig. 7(B)], as well as in and around the ventral midline [e.g. Fig. 7(D), lower]. In addition, the number of lateral CNS cells positive for TUNEL staining was sometimes increased in methanol-exposed embryos, but not as significantly as in the midline and midline-adjacent lateral regions.

To further assess the contribution of apoptosis to the late CNS defects observed in methanol-exposed embryos, we asked how methanol exposure throughout embryogenesis affects CNS development in Df(3L)H 99 embryos with a deficiency that uncovers the cell death genes grim, hid, and reaper (White et al., 1994). For these analyses we focused on stage 13 and 14 embryos, because the discrepancy in CNS cell numbers between H99 and wild-type embryos becomes too pronounced after these stages (when normal programmed cell death of lateral CNS cells causes a significant reduction in CNS cell numbers; Abrams et al., 1993). We found that the absence of the cell death genes at the 57C1-2 locus in H99 embryos leads to a significant reduction in the CNS defects resulting from methanol exposure. The occurrence of abnormal BP102 staining of CNS axons resulting from methanol exposure was reduced from about $20 \%$ in wild-type embryos exposed to $4 \%$ methanol throughout development $(n=180)$, with a range of CNS defects, to less than 3\% in H99 embryos, with very mild CNS defects $(n=40)$. Figure 7 compares 

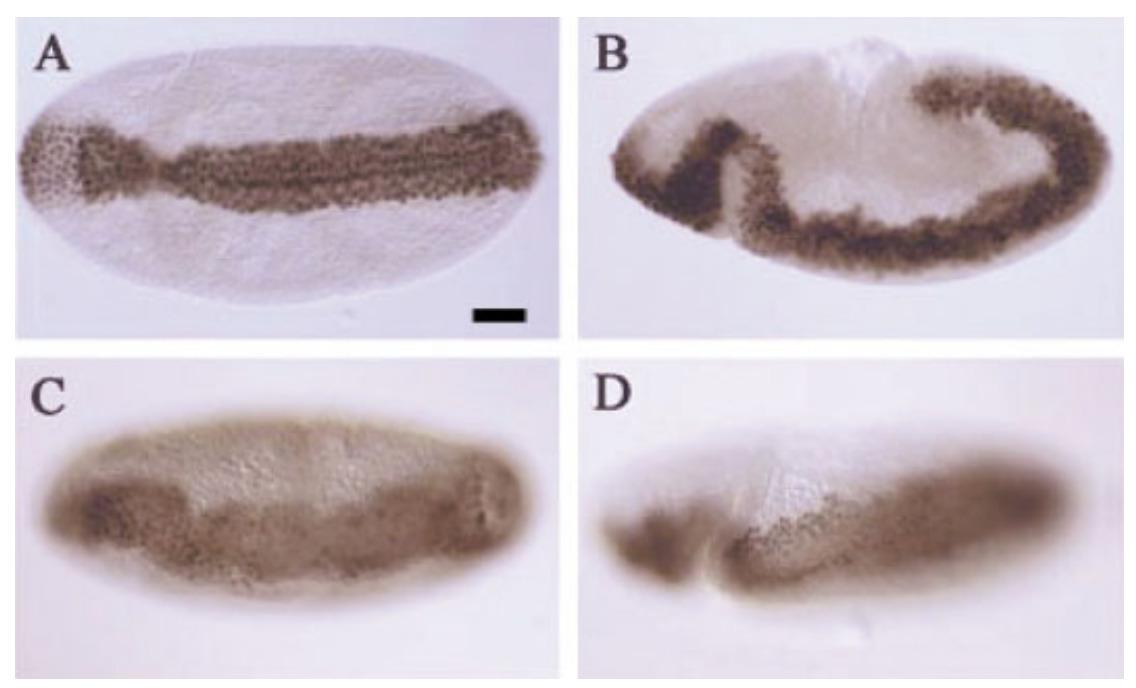

Figure 5 Aberrant distribution of the mesodermal marker, Twist, reveals defects in gastrulation and early germ band extension. (A) and (B) are control embryos, while (C) and (D) are methanolexposed embryos. Views are ventral [(A) and (C)] or lateral [(B) and (D)]. Anterior is to the right. The size bar is $50 \mu \mathrm{m}$. (A) At the completion of gastrulation Twist is expressed in the mesoderm and mesectoderm (out of focus) in a linear pattern. (B) Twist expression in a control embryo at early germ band extension. (C) In approximately $5 \%$ of embryos that were exposed to methanol from deposition, the mesodermal cells that have invaginated are positioned irregularly, indicating that gastrulation is sensitive to methanol exposure. (D) In approximately $10 \%$ of stage 8 embryos that were exposed to methanol from deposition, the mesodermal cells are twisted, suggesting that early germ band extension is also sensitive to methanol exposure.

the pattern of BP102 expression in CNS axons of wild-type embryos that developed under normal conditions or in the presence of $4 \%$ methanol relative to that seen in $\mathrm{H} 99$ embryos that developed under parallel conditions. In stage 13 embryos the commissural neurons are arranged in an X-type configuration in both wild-type and H99 embryos, because the commissures have not yet separated (Klambt and Goodman, 1991). BP102 staining levels are comparable in wild-type and H99 embryos [compare Fig. 8(A) and (B)]. Following methanol exposure throughout development, the distribution of BP102 in most H99 embryos is relatively normal, for example, Figure 8(D). In rare instances, H99 embryos have commissural defects that parallel those seen in mildly affected wild-type embryos that have been exposed to methanol throughout their development [compare Fig. 8(C) and (D)]. These results indicate that apoptosis contributes to methanol-induced neurotoxicity, but does not exclude other effects of methanol exposure during neurogenesis.

\section{DISCUSSION}

This study focuses on the detrimental effects of methanol on CNS development in Drosophila. We show that $4 \%$ methanol causes significant lethality in the Drosophila embryo, associated with severe CNS defects, including aberrant neuronal location and numbers, particularly in the thoracic and upper abdominal regions. In addition, we suggest that morphological movements are adversely affected by methanol, based on the gastrulation and germ band extension defects that we see. BrdU incorporation studies indicate that the division of neural precursors is not significantly affected by methanol. However, we found that abnormal cell movement and apoptosis contribute significantly to the dorsal displacement of the longitudinal connectives and to the commissural defects seen in methanol-exposed embryos. H99 embryos, lacking the cell death genes, grim, hid, and reaper, have significantly reduced CNS defects resulting from methanol exposure, which parallel the weak phenotype resulting from methanol exposure throughout development.

Our interpretation of the late CNS defects resulting from methanol exposure throughout embryogenesis is that these defects result primarily from aberrant cell movement during gastrulation and germ band extension. Because the ventral midline is not correctly formed, the CNS is particularly compromised during germ band retraction, which causes varying degrees of apoptosis, particularly in ventral midline cells. As 

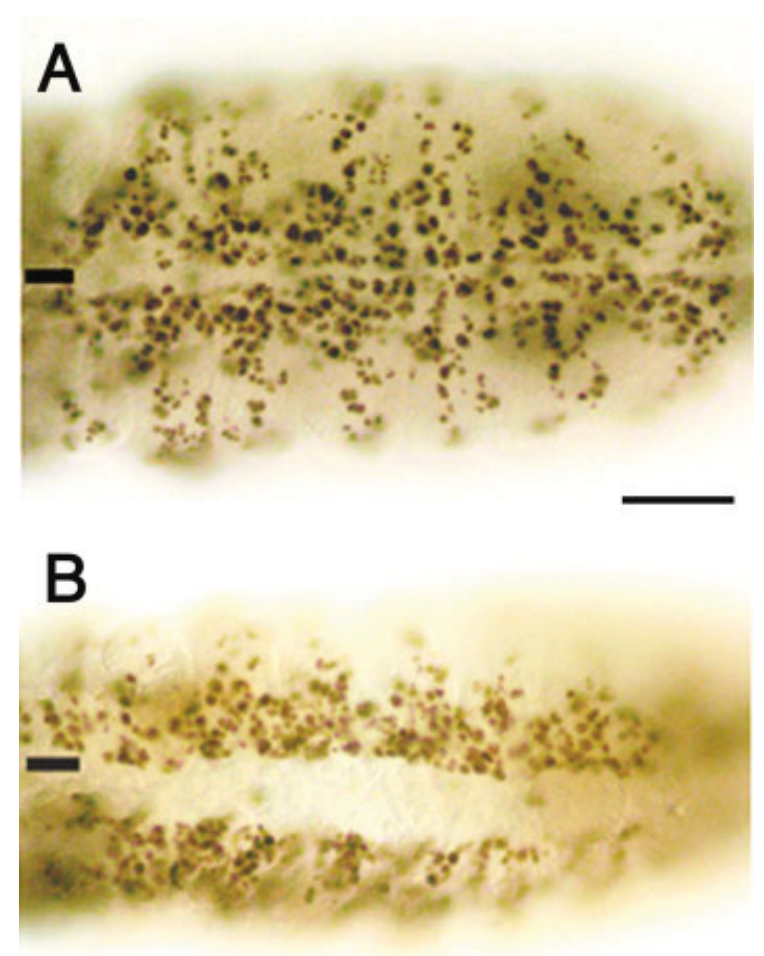

Figure 6 Replicating cells are abnormally positioned in germ band retracting embryos that developed in the presence of methanol. (A) and (B) Ventral views of a control late stage 12 embryo and an embryo that developed in the presence of $4 \%$ methanol labeled with BrdU to identify replicating cells. Anterior is to the right. Line indicates ventral midline. The size bar is $50 \mu \mathrm{m}$. (A) Normal distribution of S-phase cells. Lateral CNS cells are dividing rapidly, in addition to lateral ectodermal cells that likely include PNS cells. (B) The replicating lateral CNS cells are abnormally located adjacent to the expanded CNS ventral midline.

a result, a gap or hole forms. The essential role of the ventral midline (the fly floorplate equivalent) in CNS development and axonal pathfinding is well documented (e.g., Golembo et al., 1996).

Our finding that methanol induces apoptosis in the Drosophila embryonic CNS agrees with the finding of Hiura et al. (2000), who reported that methanol exposure causes apoptosis in tissue culture cells. The data we present here in the Drosophila embryo also agree with, and extend, studies in vertebrate embryos showing that methanol is a reproductive and developmental toxicant. Developmental toxicity has been demonstrated in vitro for rat and mouse embryos in whole embryo culture. Andrews et al. (1993, 1995, 1998) reported that explanted rat embryos cultured in the presence of $8-16 \mathrm{mg} \mathrm{MeOH} / \mathrm{mL}$ in rat serum for $24 \mathrm{~h}$ and then transferred to rat serum alone for $24 \mathrm{~h}$ had a decrease in somite number, head length, neural tube defects, and ocular lesions. Many of these embryonic defects seen in methanol-exposed vertebrate embryos are associated with abnormalities in morphogenic movements, which could involve multiple pathways. We also found that methanol exposure results in aberrant morphogenesis in the Drosophila embryo.

The exact lethal dose of methanol for humans is not known. The overall mortality of methanol poisoning is approximately $20 \%$, and among survivors the rate of permanent visual impairment is $20-25 \%$. Other complications of severe methanol intoxication in humans include coma, seizures, blindness, oliguric renal failure, cardiac failure, and pulmonary edema. Death is usually associated with terminal opisthotonos and convulsions, symptoms of severe CNS involvement and compromise (Becker, 1983). In humans methanol is oxidized by alcohol dehydrogenase to form formaldehyde and formic acid. Acidosis, attributable to the formic acid produced when methanol is metabolized, is considered to be one of the major causes of methanol toxicity (Becker, 1983). The fruit fly has two alcohol dehydrogenase enzymes, and thus likely oxidoreduces methanol in a manner paralleling that seen in humans.

That methanol can be lethal for the developing fetus and young human babies is manifested in a recent report that six Egyptian infants between the ages of 2 and 4 months died from encephalopathy, following exposure to "red alcohol," a solution containing 90\% methanol (Darwish et al., 2002). Methanol was absorbed through the skin and possibly the lungs from compresses covering sites of vaccination. The compresses were used for long periods of time. All six children became drowsy or unresponsive and methanol toxicity was considered as the cause of death in the affected children, despite the absence of both postmortem examinations and retained blood specimens from hospital laboratory tests (Darwish et al., 2002). Percutaneous methanol exposure with fatal outcome was reported in 1968 for an additional 21 children, where methanol compresses were used to treat abdominal pain. Twelve out of twenty-one children receiving methanol compresses died, compared to $2 / 27$ receiving only ethanol compresses. Thirteen of the children receiving methanol had severe respiratory depression, 14 went into coma, 11 had seizures, and seven had anuria or severe oliguria (Giminez et al., 1968).

To fully assess the risk of candidate neurotoxins to the developing fetus and to infants a range of approaches should be available that compliment each other. Obviously, risk assessment in developing mammalian embryos is the most relevant. However, it is not technically feasible to test the range of chemicals 

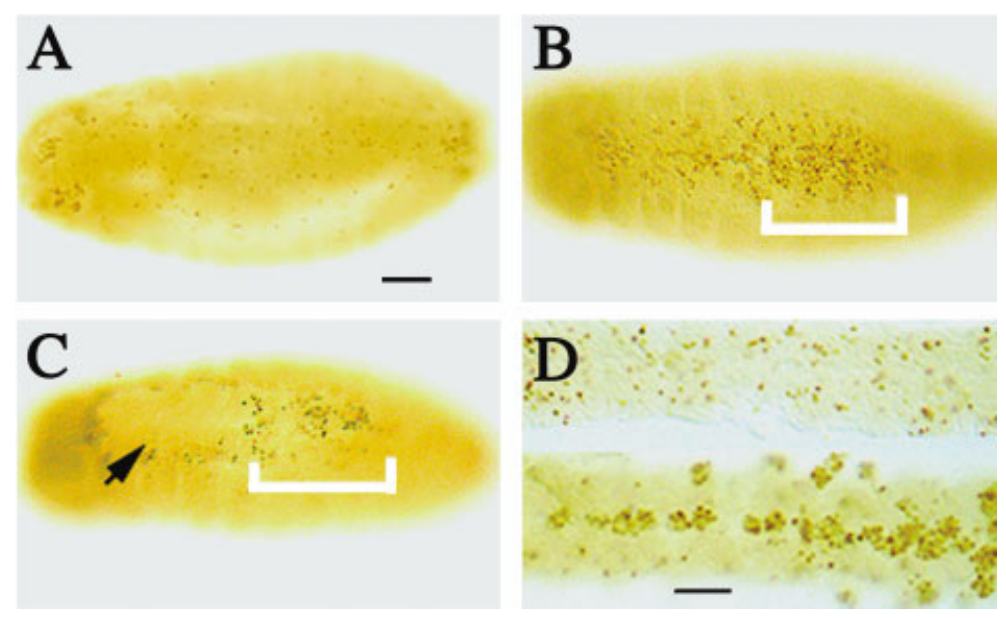

Figure 7 Methanol exposure induces inappropriate apoptosis in mature CNS cells, but not neuroblasts. TUNEL staining of stage 13-14 embryos that developed under normal conditions [(A) and (D), upper], or in the presence of $4 \%$ methanol [(B), (C), and (D), lower]. Anterior is to the left. All ventral views. The size bar is $50 \mu \mathrm{m}$. (A) Wild-type TUNEL pattern showing significant apoptosis in the brain lobes and the end of the germ band, and lower levels in the CNS and ectoderm. (B) Similarly aged methanol-sensitive embryo to (A) showing extensive ectodermal apoptosis, particularly in the upper abdominal segments, which is highlighted by the white bar. (C) Another stage 13 embryo showing an increased distribution of apoptotic cells in the abdominal regions of the CNS (white bar). Note the presence of an ectodermal hole in the thoracic region (arrow). [(D) upper] A normal stage $13 \mathrm{CNS}$ showing a somewhat randomized distribution of apoptotic nuclei. [(D) lower] A similarly aged CNS from a methanol-exposed embryo showing extensive clusters of apoptotic bodies overlying the ventral midline.

and chemical mixtures that may be neurotoxic to the developing embryo. Although examining whole vertebrate embryos that were cultured in the presence of candidate developmental neurotoxins for defects is exceptionally worthwhile, this too is an impractical screening tool. The highly accessible, relatively simple Drosophila embryo, which is sufficiently sophisticated that it may help identify developmental neurotoxins, should be considered as a screening tool to complement other neurotoxicity models. One limitation of doing neurotoxicity studies in Drosophila embryos is the variation in phenotypes caused by methanol exposure. This may be in part due to variations in the position of the egg relative to the toxin; however, it should be noted that even in the case of mutant phenotypes there is a lot of penetrance variation, which is neither well understood nor appreciated. Despite this variation our data point to a role of the
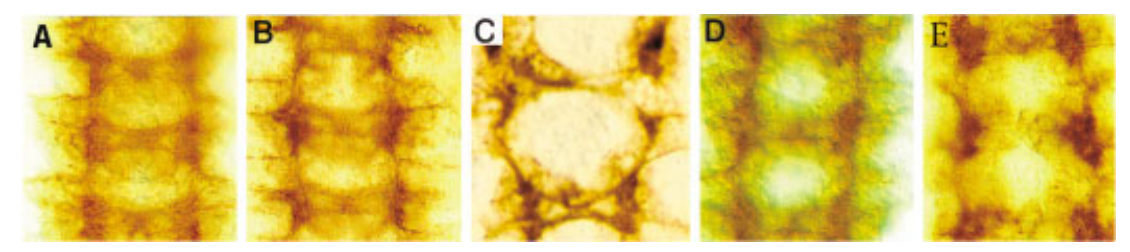

Figure 8 Embryos lacking the cell death genes grim, hid, and reaper have a low level of commissural defects due to methanol exposure. (A) and (C) are a region of the dissected CNS from a wild-type embryo that developed normally (A) or in the presence of $4 \%$ methanol (C). (B), (D), and (E) are similar regions of the dissected CNS from a H99 embryo that developed normally (B) or in the presence of 4\% methanol [(D) and (E)]. (A) and (B) At stage 13, BP102 staining is not significantly different in wild-type and H99 embryos. (C) The wild-type embryo with moderate sensitivity to methanol has defects in its commissures, and a reduction in the level of BP102 staining relative to (A). (D) Most $\mathrm{H} 99$ embryos that develop in the presence of methanol have a relatively normal CNS. (E) Although the levels of staining in (B) and (D) are comparable, commissural formation is often affected by methanol in H99 embryos. 
embryonic CNS of the fruit fly in unraveling the mechanisms of methanol neurotoxicity, and perhaps other, but not all, developmental neurotoxins.

Sincere thanks to Jay Uhler, Zhongxin Yu, and Greg Dressler for critical comments on this manuscript. Thanks also to Sophie Laio and Ken Hendrick for their attempts to set up the TUNEL assay. Thanks to the Hybridoma Core at the University of Iowa for supplying us with many of the monoclonal antibodies used in this study. We are grateful to the Bloomington stock center for providing the fly lines used. Heather Liu was partially funded by an NSF program project grant to the University of Michigan for undergraduates.

\section{REFERENCES}

Abrams JM, White K, Fessler LI, Steller H. 1993. Programmed cell death during Drosophila embryogenesis. Development 117:29-43.

Ames BN, Lee FD, Durston WE. 1973. An improved bacterial test system for the detection and classification of mutagens and carcinogens. Proc Natl Acad Sci USA 70:782-786.

Andrews JE, Ebron-McCoy M, Kavlock RJ, Rogers JM. 1995. Developmental toxicity of formate and formic acid in whole embryo culture: a comparative study with mouse and rat embryos. Teratology 51:243-251.

Andrews JE, Ebron-McCoy M, Logsdon TR, Mole LM, Kavlock RJ, Rogers JM. 1993. Developmental toxicity of methanol in whole embryo culture: a comparative study with mouse and rat embryos. Toxicology 81:205-215.

Andrews JE, Ebron-Mccoy M, Schmid JE, Svendsgaard D. 1998. Effects of combinations of methanol and formic acid on rat embryos in culture. Teratology 58:54-61.

Arendt D, Nubler-Jung K. 1999. Comparison of early nerve cord development in insects and vertebrates. Development 126:2309-2325.

Becker CE. 1983. Methanol poisoning. J Emerg Med 1:51-58.

Carroll SB, DiNardo S, O'Farrell PH, White RA, Scott, MP. 1998. Temporal and spatial relationships between segmentation and homeotic gene expression in Drosophila embryos: distributions of the fushi tarazu, engrailed, sex combs reduced, Antennapedia, and Ultrabithorax proteins. Genes Dev 2:350-360.

Cornell RA, Von Ohlen T. 2000. Vnd/nkx, ind/gsh, and $\mathrm{msh} / \mathrm{msx}$ : conserved regulators of dorsoventral neural patterning? Curr Opin Neurobiol 10:63-71.

Darwish A, Roth CE, Duclos P, Ohn SA, Nassar A, Mahoney F, Vogt R, Arthur RR. 2002. Investigation into a cluster of infant deaths following immunization: evidence for methanol intoxication. Vaccine 20:3585-3589.

Dauer W, Przedborski S. 2003. Parkinson's disease: mechanisms and models. Neuron 39:889-909.

Frasch M, Hoey T, Rushlow C, Doyle H, Levine M. 1987. Characterization and localization of the even-skipped protein of Drosophila. EMBO J 6:749-759.

George-Weinstein M, Foster RF, Gerhart JV, Kaufman SJ. 1993. In vitro and in vivo expression fo alpha 7 integrin and desmin define the primary and secondary myogenic lineages. Dev Biol 156:209-229.

Giminez ER, Vallejo NE, Roy E, Los M, Izurieta EM, Ross1 S. 1968. Percutaneous alcohol intoxication. Clin Toxicol 1:39-48.

Golembo M, Raz E, Shilo BZ. 1996. The Drosophila embryonic midline is the site of Spitz processing, and induces activation of the EGF receptor in the ventral ectoderm. Development 122:3363-3370.

Goodman CS, Bastiani MJ, Doe CQ, du Lac S, Helfand SL, Kuwada JY, Thomas JB. 1984. Cell recognition during neuronal development. Science 225:1271-1279.

Halter DA, Urban J, Rickert C, Ner SS, Ito K, Travers AA, Technau GM. 1995. The homeobox gene repo is required for the differentiation and maintenance of glia funciton in the embryonic nervous system of Drosophila melanogaster. Development 121:317-332.

Hiura TS, Li N, Kaplan R, Horwitz M, Seagrave JC, Nel AE. 2000. The role of a mitochondrial pathway in the induction of apoptosis by chemicals extracted from diesel exhaust particles. J Immunol 165:2703-2711.

Jacobs JR. 2000. The midline glia of Drosophila: a molecular genetic model for the developmental functions of glia. Prog Neurobiol 62:475-508.

Klambt C, Goodman CS. 1991. Role of the midline glia and neurons in the formation of the axon commissures in the central nervous system of the Drosophila embryo. Ann NY Acad Sci 633:142-159.

Liu JJ, Daya MR, Mann NC. 1999. Methanol-related deaths in Ontario. J Toxicol Clin Toxicol 37:69-73.

Mellerick DM, Kassis JA, Zhang SD, Odenwald WF. 1992. castor encodes a novel zinc finger protein required for the development of a subset of CNS neurons in Drosophila. Neuron 9:789-803.

Patel NH, Schafer B, Goodman CS, Holmgren R. 1989. The role of segment polarity genes during Drosophila neurogenesis. Genes Dev 3:890-904.

Rothberg JM, Jacobs JR, Goodman CS, Artavanis-Tsakonas S. 1990. slit: an extracellular protein necessary for development of midline glia and commissural axon pathways contains both EGF and LRR domains. Genes Dev 4:2169-2187.

Shermoen AW. 2000. BrdU Labeling of Chromosomes. In Drosophila Protocols. Sullivan, Ashburner, Hawley, editors. New York: Cold Spring Harbor Laboratory Press, p 57-65.

Sweeney ST, Hidalgo A, de Belle JS, Keshishian H. 2000. Functional Cell Ablation. In Drosophila Protocols. Sullivan, Ashburner and Hawley, editors. New York: Cold Spring Harbor Laboratory Press, p 449-477.

Thisse BSC, Gorostiza-Thisse C, Perrin-Schmitt F. 1988. Sequence of the twist gene and nuclear localization of its protein in endomesodermal cells of early Drosophila embryos. EMBO J 7:2175-2183.

US Environmental Protection Agency. 1994. Wshington, DC. Toxics release inventory-public data. EPA-745-R-94-001.

White K, Grether ME, Abrams, JM, Young L, Farrell K, Steller H. 1994. Genetic control of programmed cell death in Drosophila. Science 264:677-683. 